\title{
Detection of Calcium Transients in Drosophila Mushroom Body Neurons with Camgaroo Reporters
}

\author{
Dinghui Yu, ${ }^{1}$ Geoffrey S. Baird, ${ }^{3}$ Roger Y. Tsien, ${ }^{3,4}$ and Ronald L. Davis ${ }^{1,2}$ \\ Departments of ${ }^{1}$ Molecular and Cellular Biology and ${ }^{2}$ Psychiatry and Behavioral Sciences, Baylor College of Medicine, Houston, Texas 77030, and \\ ${ }^{3}$ Department of Pharmacology, ${ }^{4}$ Howard Hughes Medical Institute, University of California, San Diego, San Diego, California 92093
}

\begin{abstract}
Camgaroos are yellow fluorescent protein derivatives that hold promise as transgenically encoded calcium sensors in behaving animals. We expressed two versions of camgaroo in Drosophila mushroom bodies using the galactosidase-4 (GAL4) system. Potassium depolarization of brains expressing the reporters produces a robust increase in fluorescence that is blocked by removing extracellular calcium or by antagonists of voltage-dependent calcium channels. The fluorescence increase is not attributable to cytoplasmic alkalization; depolarization induces a slight acidification of the cytoplasm of mushroom body neurons. Acetylcholine applied near the dendrites of the mushroom body neurons induces a rapid and ipsilateral-specific fluorescence increase in the mushroom body axons that is blocked by antagonists of calcium channels or nicotinic acetylcholine receptors. Fluorescence was observed to increase in all three classes of mushroom body neurons, indicating that all types respond to cholinergic innervation.
\end{abstract}

Key words: mushroom bodies; calcium; optical imaging; Drosophila; camgaroos; GFP

\section{Introduction}

The central role of calcium in neuronal signaling and physiology (Zucker, 1999; Augustine, 2001; Bootman et al., 2001; Rose and Konnerth, 2001; West et al., 2001) has stimulated intense interest in monitoring and understanding the complex quantitative, spatial, and temporal components of calcium signaling. Calcium levels in neurons have been monitored using several different imaging procedures. Optical detection of changes in calcium concentration with ion-sensitive fluorescent dyes provides for robust temporal and spatial resolution of calcium dynamics. For instance, dyes such as fura- 2 or calcium green have been used to monitor localized calcium increases in brain slices attributable to subthreshold EPSPs, as well as cell-wide increases in calcium from the propagation of action potentials (Yuste and Denk, 1995). Nevertheless, the utility of these dyes can be limiting. The tissue must be bathed with cell-permeable dyes that may not penetrate complete or carefully loaded intracellularly via an electrode. Several different transgenes have also been fashioned to encode reporters of calcium dynamics. Aequorin, a photoprotein from the jellyfish, Aequorea victoria, displays calcium-sensitive chemiluminescence when supplied with a hydrophobic prosthetic group, coelenterazine (Chiesa et al., 2001). Although aequorin offers a large dynamic range, less than one photon is generated per aequorin molecule, so that the bioluminescence is very dim and hard to image with good spatial resolution. Moreover, the aequorin can be exhausted during the production of signal, and the cofactor coelenterazine must be supplied exogenously.

Received Aug. 5, 2002; revised 0ct. 11, 2002; accepted 0ct. 14, 2002.

This work was supported by National Institutes of Health (NIH) Grant NS19904, the Mathers Charitable Trust, and the R.P. Doherty-Welch Chair in Science (R.L.D.). R.Y.T. and G.S.B. were supported by NIH Grant NS27177 (R.Y.T.) and the Howard Hughes Medical Institute. We thank Drs. Gregg Roman and Yuzhong Cheng for critically reading this manuscript.

Correspondence should be addressed to Ronald L. Davis, Department of Molecular and Cellular Biology, Baylor College of Medicine, Houston, TX 77030. E-mail: rdavis@bcm.tmc.edu.

Copyright $\odot 2002$ Society for Neuroscience $\quad$ 0270-6474/02/220064-09\$15.00/0
The cameleons are hybrid proteins consisting of calmodulin and a calmodulin-binding peptide flanked by cyan and yellow mutants of green fluorescent protein (GFP). Absorption of light by the cyan fluorescent protein can lead to energy transfer to the yellow fluorescent protein (YFP). The efficiency of such energy transfer increases when elevated calcium causes the calmodulin to fold around the concatenated peptide (Miyawaki et al., 1997; Griesbeck et al., 2001). Although the cameleons have been used to monitor calcium levels in the pharyngeal muscle and neurons of transgenic Caenorhabditis elegans (Kerr et al., 2000), the $\mathrm{Ca}^{2+}$-induced enhancement in energy transfer efficiency is modest, so that the dynamic range in ratio of yellow-to-cyan emissions is currently at most a factor of two in vitro and considerably less in vivo.

The camgaroos (Baird et al., 1999; Griesbeck et al., 2001) and similar molecules (Nagai et al., 2001; Nakai et al., 2001) are the newest proteins engineered as fluorescent calcium indicators. Camgaroo-1 was constructed as an insertion of Xenopus calmodulin at residue Y145 of enhanced YFP (EYFP) (Baird et al., 1999). The fluorescence of the fusion protein is sensitive to calcium concentrations in transfected cells with an apparent $K_{\mathrm{d}}$ of $7 \mu \mathrm{M}$. Fluorescence increases of up to sevenfold in vitro result from calcium-induced deprotonation of the EYFP chromophore. Camgaroo-2 is identical in structure to camgaroo-1 but was isolated after random mutagenesis of camgaroo- 1 in a screen for brighter fluorescence (Griesbeck et al., 2001). The $K_{\mathrm{d}}$ for calcium is similar to that of camgaroo-1; camgaroo-2 also has a similar fluorescence enhancement of sixfold to sevenfold. The alteration of camgaroo-2 that is responsible for the increased basal fluorescence is an amino acid change at residue 69 from glutamine to methionine (Q69M). Camgaroo-1 and camgaroo-2 have only been tested in transfected cells in culture, not yet in complex tissues or intact organisms.

We used the Drosophila galactosidase-4 (GAL4) system to drive the expression of the camgaroos in the mushroom bodies as the first step toward developing a system for monitoring calcium 
levels in the fly brain. Our experiments on isolated brains expressing these indicators show that they indeed report changes in calcium concentration, and this report can be obtained from cell bodies, dendrites, or axons of neurons. We also used these indicators to demonstrate for the first time that all three types of mushroom body neurons respond to the neurotransmitter acetylcholine (ACh).

\section{Materials and Methods}

Molecular biology and genetics. Complementary DNAs encoding camgaroo-1 and -2 were inserted separately into the NotI site of the upstream activating sequence (UAS) vector pPBretU-H/X (Roman et al., 1999). The constructs were verified by restriction mapping and sequencing and used to transform Drosophila. Five independent transformants of camgaroo-1 (P\{UAS-camgaroo-1\}) and 10 independent transformants of camgaroo-2 (P\{UAS-camgaroo-2\}) were obtained and mapped genetically. For the $\mathrm{P}\{$ UAS-camgaroo- 1$\}$ transgenic lines, one maps to X chromosome, two map to second chromosome, and two map to the third chromosome. For the P\{UAS-camgaroo- 2$\}$ transgenic lines, six map to the second chromosome and four map to the third chromosome.

The P\{UAS-camgaroo\} transgenic flies were crossed with the GAL4 line 238Y (Yang et al., 1995). Progeny flies containing both the P $\{$ UAScamgaroo $\}$ transgene, and the 238Y GAL4 transgene were used for all experiments. Flies were maintained on a $12 \mathrm{hr}$ light/dark cycle on standard Drosophila medium at $24^{\circ} \mathrm{C}$.

Brain dissections and imaging. Fly brains were dissected from the head capsule in HL3 solution with $1.5 \mathrm{~mm} \mathrm{CaCl}_{2}$ (Stewart et al., 1994) unless otherwise specified and secured to the bottom of a small Petri dish. The brains were imaged using a Leica (Nussloch, Germany) TCS confocal microscope with a $488 \mathrm{~nm}$ excitation line and with emitted light collected from $520 \pm 15 \mathrm{~nm}$.

For potassium depolarization experiments, the brains were scanned at the speed of one frame per $2.5 \mathrm{sec}$ for $100 \mathrm{sec}$. A volume of $120 \mu \mathrm{l} \mathrm{of} 3 \mathrm{M}$ $\mathrm{KCl}$ was added to $5 \mathrm{ml}$ of HL3 at $40 \mathrm{sec}$ to give a final potassium concentration of $77 \mathrm{~mm}$. The baseline fluorescence $\left(F_{0}\right)$ was calculated as the average fluorescence from 0 to $37 \mathrm{sec}$ for the region of interest. The percentage change in fluorescence for graphical presentation was calculated from the following expression: $\left(F-F_{0}\right) / F_{0} \times 100$. Because of bleaching that generally occurred early in some scans, the change in fluorescence was calculated as the highest average fluorescence intensity over the region of interest observed during the response period divided by the average fluorescence over the region of interest before depolarization (from 20 to $37 \mathrm{sec}$ ). Diltiazem (diltiazem hydrochloride; Sigma, St. Louis, MO) and verapamil ( \pm -verapamil hydrochloride; Sigma) were added 10-15 min before collecting images. For the ionomycin experiments, ionomycin (ionomycin calcium salt; Sigma) was added to brains in culture medium without calcium at $1 \mathrm{~min}$. Calcium was added at 1.5 min and EGTA at 5 min.

Intracellular $\mathrm{pH}$ changes were followed using nigericin (nigericin sodium salt; Fluka, Neu-Ulm, Germany) (Boyarsky et al., 1988) along with the membrane-permeable and ratiometric $\mathrm{pH}$ indicator SNARF-1 (Molecular Probes, Eugene, OR). SNARF-1 $(50 \mu \mathrm{g})$ was dissolved in $20 \mu \mathrm{l} \mathrm{of}$ DMSO containing $10 \%$ pluronic acid and further diluted to $1.6 \mathrm{ml}$ with HL3 solution. Dissected brains were loaded with SNARF-1 at a final concentration of $50 \mu \mathrm{M}$ for $1 \mathrm{hr}$ and used for potassium depolarization experiments or to follow fluorescence change as a function of intracellular $\mathrm{pH}$. To follow intracellular $\mathrm{pH}$, SNARF-1-loaded brains were treated with $130 \mathrm{mM} \mathrm{K}^{+}$of varying $\mathrm{pH}$ values and containing the proton ionophore nigericin $(10 \mu \mathrm{g} / \mathrm{ml})$. Extracellular $\mathrm{pH}$ after these treatments reflects the intracellular $\mathrm{pH}$. The change of the SNARF-1 fluorescence ratio $(660 \pm 20: 580 \pm 20 \mathrm{~nm})$ is an indicator of intracellular $\mathrm{pH}$ change and was followed simultaneously with camgaroo fluorescence $(520 \pm 15 \mathrm{~nm})$.

ACh (acetylcholine chloride; Sigma) was applied for $2 \mathrm{sec}$ with a puffer pipette driven by a Picospritzer II (General Valve, Fairfield, NJ) at a pressure of 2-3 psi. The delivery pipette had an internal diameter at the opening of $0.5 \mu \mathrm{m}$, and the tip was placed within $5 \mu \mathrm{m}$ of the brain just anterior to the calyx. Placement of the pipette within the calyx produced motion artifacts during ACh delivery. Baseline fluorescence $\left(F_{0}\right)$ for the graphical representation of experiments shown in Figure 6 was the average fluorescence from 0 to $17 \mathrm{sec}$ before the application of $\mathrm{ACh}$, and the percentage change was calculated as described above. To determine the average increase in fluorescence for camgaroo-1, the highest fluorescence value for the region of interest obtained after ACh application was divided by the average fluorescence for the region obtained before application (from 10 to $17 \mathrm{sec}$ ). Because of the significant bleaching that occurred early in the scans displayed in Figure $7, F_{0}$ was calculated as the average fluorescence for the region of interest across the whole scan. The average increase was calculated using only the average fluorescence immediately before ACh application and the highest average value within 3 sec after application. The ACh receptor antagonists tubocurine ( $d$ tubocurarine chloride; Sigma) and bungarotoxin ( $\alpha$-bungarotoxin; Sigma) and the L-type calcium channel blockers were added to the incubation medium after an initial scan, and the camgaroo response was retested $10-15$ min later.

\section{Results}

\section{Expression of the camgaroos in the mushroom bodies}

We used the GAL4 system to express camgaroo-1 or camgaroo-2 in the mushroom bodies of the adult brain. Mushroom bodies are conspicuous structures in the brain that function in olfactory learning (Roman and Davis, 2001). The mushroom bodies comprise several thousand neurons with the neuronal cell bodies residing on the dorsal posterior cortex of the brain. Their dendritic processes extend ventrally into a spherical neuropil structure termed the calyx in which they receive olfactory information from the antennal lobes. One class of mushroom body neuron extends axons as a bundle to the anterior face of the brain where the axons split into a vertically oriented and mace-shaped area of neuropil known as the $\alpha$ lobe and a horizontally oriented and mace-shaped area of neuropil known as the $\beta$ lobe. A second type of mushroom body neuron extends an axon that splits into neuropil areas known as the $\alpha^{\prime}$ and $\beta^{\prime}$ lobes. These neuropil areas are shaped and oriented like the $\alpha$ and $\beta$ lobes. A third type of mushroom body neuron fails to split and sends a single extension into the horizontally oriented $\gamma$ lobe. Thus, there are three different classes of mushroom body cells termed $\alpha / \beta, \alpha^{\prime} / \beta^{\prime}$, and $\gamma$ neurons (Crittenden et al., 1998).

Complementary DNAs for camgaroo- 1 and camgaroo-2 were cloned into a P-factor vector adjacent to tandem UAS sequences.
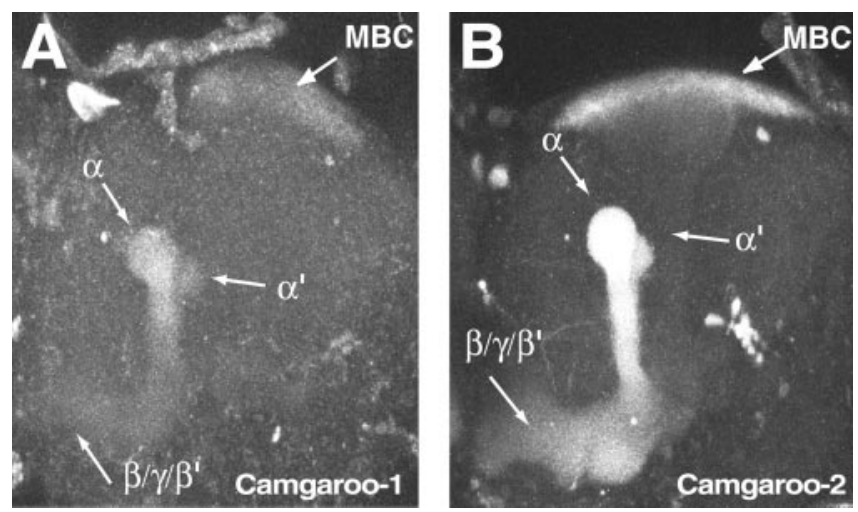

Figure 1. Basal expression of camgaroo in the mushroom bodies. A, A maximum projection image of a portion of a dissected brain showing camgaroo- 1 expression in the mushroom bodies from the GAL4 driver 238Y. This image was collected in the absence of any stimulation. This frontodorsal view reveals the cell bodies of mushroom body neurons $(M B C)$, the dorsally oriented $\alpha$ and $\alpha^{\prime}$ lobes, and the medially oriented $\beta, \beta^{\prime}$, and $\gamma$ lobes. The $\beta, \beta^{\prime}$, and $\gamma$ lobes are not distinguishable from each other and are located deep in the brain. $B, A$ similar image to that in $A$ showing the more robust expression of camgaroo- 2 with the same GAL4 driver. A halo of faint expression of camgaroo- 2 is also observed in the calyx ( $C$ ). The image of camgaroo-2 was collected at a lower photomultiplier tube voltage. 

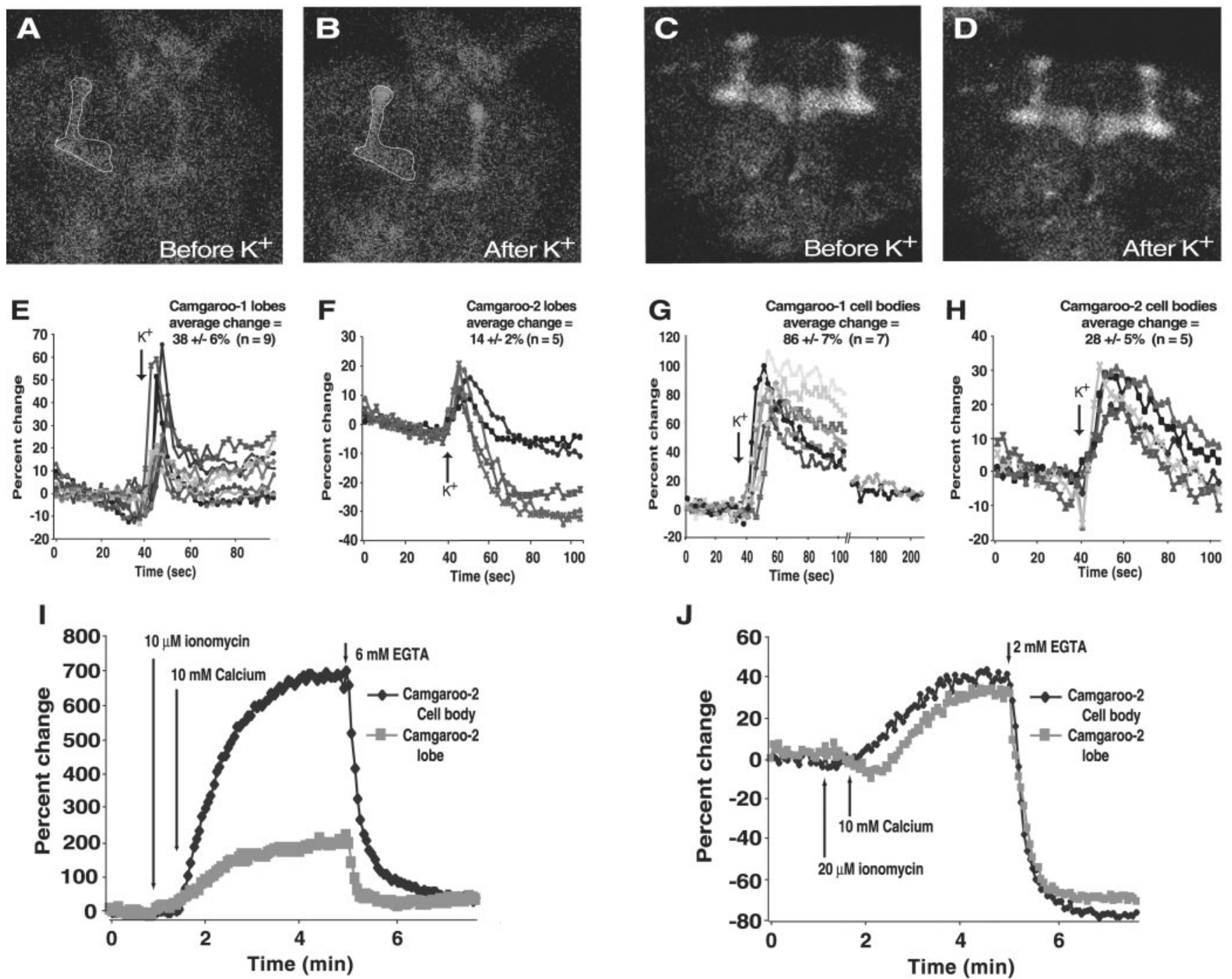

Figure 2. Increase of camgaroo fluorescence with potassium depolarization and calcium influx. The camgaroo fluorescence from the mushroom bodies in dissected brains was monitored by confocal scanning microscopy. At 40 sec into the scan for $E-H$ (arrows), concentrated potassium was added to the incubation medium to a final concentration of $77 \mathrm{~mm}$. For these panels, the baseline fluorescence was established by averaging the intensity from 0 to $37 \mathrm{sec}$ of each scan. The percentage change is the increase observed at the highest point of each scan relative to the average fluorescence from 20 to $37 \mathrm{sec}$ of each scan. For all panels, one trace represents one brain. $A, B$, Camgaroo-1 fluorescence in the lobes before $(A)$ and after $(B)$ potassium depolarization. Camgaroo-1 fluorescence is very weak: the mushroom body lobes of the right hemisphere of the fly brain are outlined. C, D, Camgaroo-2 fluorescence before $(C)$ and after $(D)$ potassium depolarization. Basal camgaroo-2 fluorescence is more robust than camgaroo-1, but the increase of fluorescence with potassium depolarization is not obvious from direct visual inspection. $E$, A family of traces representing the change in fluorescence in the lobes of brains expressing camgaroo-1. A robust increase is fluorescence is observed within seconds after potassium depolarization, which occurred at 40 sec into the scan. $F$, A family of traces representing the change in fluorescence in the lobes of brains expressing camgaroo-2. The increase in fluorescence with potassium depolarization at $40 \mathrm{sec}$ is less robust than that observed for camgaroo- 1 but is still significant. This reporter also bleaches much more rapidly than camgaroo-1 (see also $H$ ). $G, A$ family of traces representing the change in fluorescence in the cell bodies of brains expressing camgaroo-1. A robust increase in fluorescence is observed within seconds after potassium depolarization, which occurred at $40 \mathrm{sec}$ into the scan. The responses of two brains were followed to estimate the time to return to baseline, which occurred at $\sim 160 \mathrm{sec}$ after depolarization. $H$, A family of traces representing the change in fluorescence in the cell bodies of brains expressing camgaroo-2. The increase in fluorescence in the cell bodies with potassium depolarization at 40 sec is less robust than that observed for camgaroo-1. I, A trace of the fluorescence emission of camgaroo- 1 in the cell bodies or lobes after treatment with ionomycin. A 700 and $200 \%$ increase in fluorescence occurred during addition of calcium in the cell bodies and lobes, respectively. This increase was reversed by EGTA.J, A trace of the fluorescence emission of camgaroo-2 in the cell bodies or lobes after treatment with ionomycin. A 40 and 30\% increase in fluorescence occurred during addition of calcium in the cell bodies and lobes, respectively. This increase was reversed by EGTA.

Several independent transgenic lines for P\{UAS-camgaroo-1 $\}$ and P $\{$ UAS-camgaroo-2 $\}$ were obtained (see Materials and Methods). These were mapped and crossed with P\{GAL4\} enhancer detector lines that are known to express GAL4 in adult mushroom bodies. Freshly dissected brains from P $\{$ GAL4 $\} / \mathrm{P}\{$ UAS-camgaroo $\}$ doubleheterozygous animals were mounted in culture medium and visualized by laser-scanning confocal microscopy.

The brains of flies expressing camgaroo-1 from the element $\mathrm{P}\{$ GAL4 $\} 238 \mathrm{Y}$ exhibited very modest fluorescence in the mushroom bodies (Fig. 1A). Fluorescence was observed in the cell bodies of the mushroom body neurons, as well as in the $\alpha, \alpha^{\prime}, \beta$, $\beta^{\prime}$, and $\gamma$ lobes, indicating that camgaroo- 1 diffuses into the axons of these neurons. No fluorescence was detectable in the mushroom body calyx. The fluorescence from camgaroo- 2 was much more intense, even under more stringent visualization settings (Fig. $1 B$; compare background fluorescence of $A$ and $B$ ). Each lobe neuropil and the cell body region exhibited bright fluorescence. In addition, the calyx was barely visible in flies expressing camgaroo-2. No fluorescence was detectable in the brains of P\{UAS-camgaroo $\}$ animals lacking a GAL4 driver (data not 
A
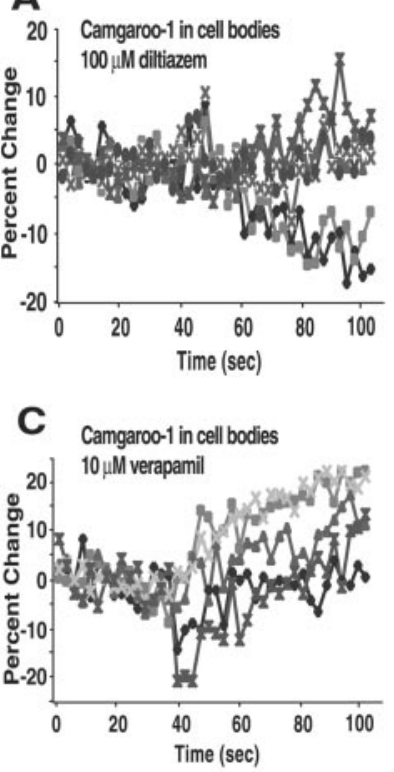

E

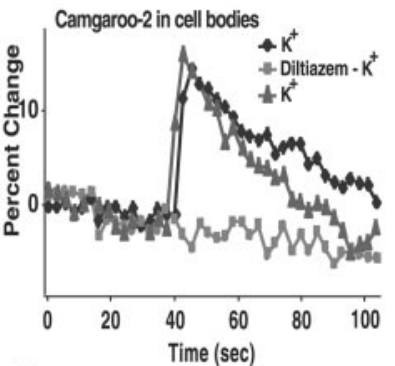

G

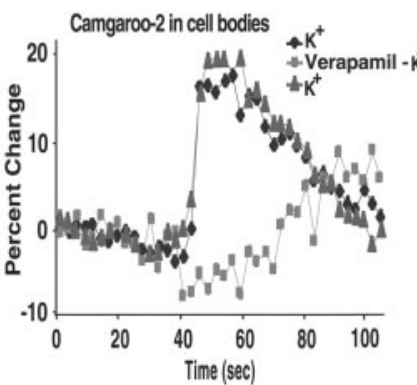

I

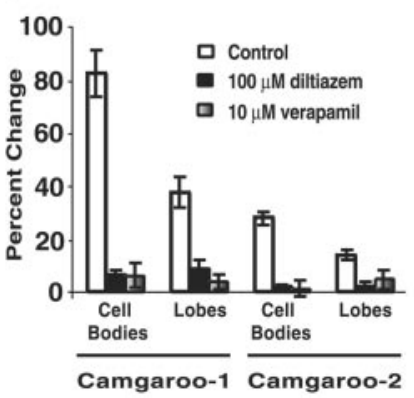

Figure 3. Rapid increases in camgaroo fluorescence with potassium depolarization are inhibited by calcium channel blockers. $A-D, A$ family of traces of the change in fluorescence is shown in $A-D$. Each trace represents one brain. At $40 \mathrm{sec}$, concentrated potassium was added to the incubation medium as in Figure 2 to bring the final concentration to $77 \mathrm{~mm}$. Calcium channel blockers were added to the incubation medium 10-15 min before imaging. Each panel presents data from five to six individual brains. $A, B$, Camgaroo- 1 fluorescence in the cell bodies $(A)$ or lobes $(B)$ in the presence of $100 \mu \mathrm{m}$ diltiazem. The rapid increase in fluorescence normally shown). Therefore, the P\{GAL4\}238Y element drives camgaroo expression rather specifically in the cell bodies and processes of adult mushroom body neurons, with camgaroo-2 fluorescence being more intense, as expected, than that of camgaroo-1.

\section{Camgaroos respond to neuronal depolarization}

To determine whether the camgaroos would respond to neuronal depolarization and calcium influx, we increased the extracellular potassium concentration in the culture medium to induce depolarization throughout the brain while scanning for fluorescence. There was an obvious increase in fluorescence visible by direct inspection that was specific to the mushroom bodies of brains expressing camgaroo-1 (Fig. 2A,B). A similar increase was not visible from brains expressing camgaroo-2 (Fig. $2 C, D$ ). This is likely attributable to a higher basal fluorescence along with a smaller quantitative response in the region of interest. Figure $2 E$ illustrates the quantitative response in the lobes of nine brains expressing camgaroo- 1 . Each brain that was imaged produced a reliable response with potassium depolarization with an average change in fluorescence of $38 \%$. The quantitative response in the lobes for camgaroo- 2 was $14 \%$, approximately one-half that observed with camgaroo-1 (Fig. $2 F)$. In addition, continued imaging of camgaroo-2-expressing brains after potassium depolarization showed a more rapid bleaching of this reporter in several of the brains that were imaged compared with camgaroo-1 (Fig. 2F). Thus, both reporters showed a rather robust response that reaches a maximum within a few seconds after depolarization, consistent with the possibility that the reporters detect an increase in intracellular calcium in the axons and axon terminals of mushroom body neurons.

The responses of camgaroo in the cell bodies of mushroom body neurons were more robust than that observed in the lobes. The quantitative response for camgaroo- 1 in the cell bodies averaged $83 \%$ (Fig. 2G); the response for camgaroo-2 in the cell bodies averaged $28 \%$ (Fig. $2 \mathrm{H}$ ). The response profiles were quite similar to those obtained from the lobes, although they took longer to return to a baseline level (Fig. 2G). Generally, the fluorescence increased immediately or within a few seconds after adding potassium and peaked $\sim 10 \mathrm{sec}$ later (Fig. $2 G, H$ ). To estimate the maximum fluorescence from the camgaroos under these expression and imaging conditions, we treated the brains in culture with the calcium ionophore ionomycin, followed by extracellular calcium. Camgaroo-1 had a dynamic range in the cell

observed with depolarization (Fig. 2) is primarily blocked by diltiazem. Similar results were obtained in the presence of diltiazem for brains expressing camgaroo-2 (data not shown). Results are summarized in I. C, D, Camgaroo-1 fluorescence in the cell bodies $(C$ or lobes $(D)$ in the presence of $10 \mu \mathrm{m}$ verapamil. The rapid increase in fluorescence normally observed with depolarization (Fig. 2) is blocked by verapamil. Similar results were obtained in the presence of verapamil for brains expressing camgaroo-2 (data not shown). Results are summarized in I. $E-H$, Camgaroo-2 fluorescence in washout experiments. A representative trace for one brain is shown in each panel. Similar results were obtained with four individual brains in each experiment. $E$, F, Camgaroo-2 fluorescence in the cell bodies $(E)$ or lobes ( $F$ ). Brains were first depolarized with high potassium, and then the medium replaced with fresh saline. Ten minutes later, the brains were depolarized with high potassium in the presence of $100 \mu \mathrm{m}$ diltiazem. The medium was replaced, and, $10 \mathrm{~min}$ later, the brains were again challenged with high potassium. Diltiazem blocked the response to potassium, but this was reversible. $G, H$, Camgaroo-2 fluorescence in the cell bodies $(G)$ or lobes $(H)$. Brains were first depolarized with high potassium, and then the medium replaced with fresh saline. Ten minutes later, the brains were depolarized with high potassium in the presence of $10 \mu \mathrm{m}$ verapamil. The medium was replaced, and, 10 min later, the brains were again challenged with high potassium. Verapamil blocked the response to potassium, but this was reversible. I, Summary plot of the effect of channel blockers on camgaroo- 1 and camgaroo-2 fluorescence in the cell bodies or lobes observed during potassium depolarization. 

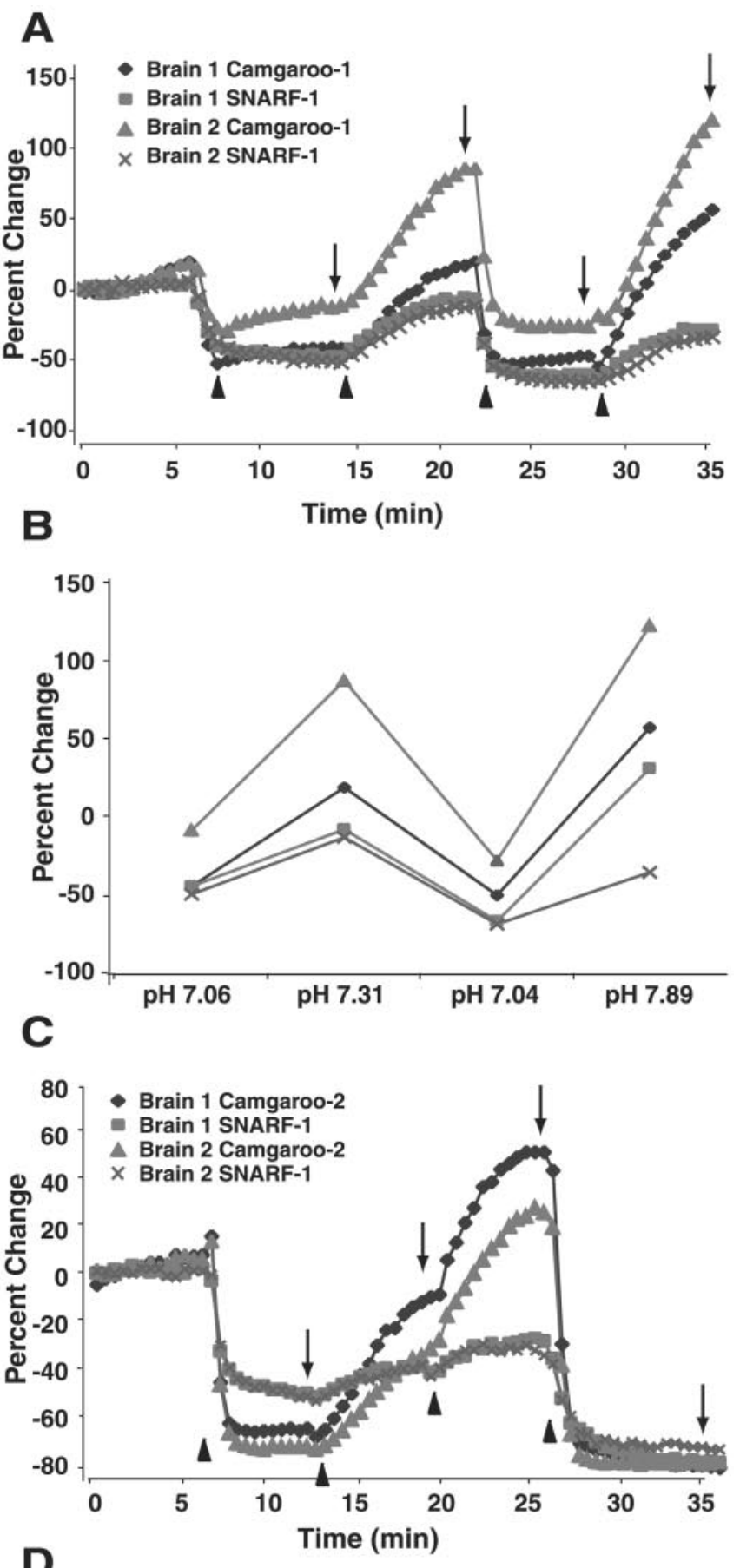

D

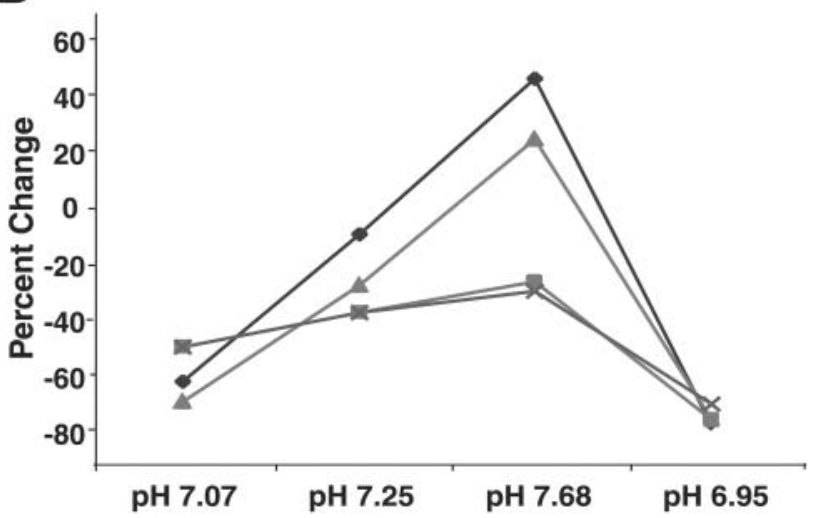

bodies of $\sim 700 \%$ under these conditions, whereas the dynamic range for camgaroo- 2 in the cell bodies was $\sim 40 \%$. Thus, the dynamic range of these reporters extends well beyond the maximum fluorescence produced by the strong stimulus of potassium depolarization.

Increases in camgaroo fluorescence require the activity of voltage-gated calcium channels

To explore the possibility that the increases in camgaroo fluorescence occur from an influx of extracellular calcium during depolarization, we depolarized brains with potassium in the presence of pharmacological blockers of voltage-gated calcium channels (Hockerman et al., 1997) and compared the responses to the reproducible increases observed in Figure 2. Diltiazem and verapmil are agents that block L-type voltage-gated channels in insect neurons at micromolar concentrations (Morales et al., 1999; Wicher et al., 2001). Diltiazem blocked the rapid fluorescence increase with potassium depolarization in the cell bodies (Fig. $3 A, E$ ) and lobes (Fig. $3 B, F$ ) of brains expressing either camgaroo-1 or camgaroo-2. Verapamil produced similar effects on the potassium depolarization-induced increase in camgaroo fluorescence (Fig. $3 C, D, G, H$ ). The block of the fluorescence response observed with the calcium channel antagonists was reversible, as demonstrated by treating brains with high potassium, then high potassium in the presence of the antagonists, and finally high potassium once again with media exchanges between each step. The initial fluorescence response observed with high potassium was completely blocked by diltiazem or verapamil during the second challenge by potassium (Fig. $3 E-H$ ). The third challenge by potassium after drug washout was nearly identical to the response observed before the antagonists were applied. The effects of calcium channel blockers are summarized in Figure 3I. These experiments suggest strongly that the increase in camgaroo- 1 and camgaroo- 2 fluorescence that occurs with depolarization is attributable to the influx of extracellular calcium through voltage-dependent calcium channels. This conclusion is supported by experiments in which the increases in fluorescence were blocked by chelating extracellular calcium (see below).

The increase in camgaroo fluorescence with depolarization is not attributable to changes in intracellular $\mathrm{pH}$

Neuronal activity is associated with changes in intracellular $\mathrm{pH}$. For example, bursting activity in the hippocampus or other neurons has been shown to produce intracellular acidification, per-

$\leftarrow$

Figure 4. Camgaroo and SNARF-1 responses to changes in intracellular pH. Brains were incubated with the membrane-permeable pH indicator SNARF-1 (50 $\mu \mathrm{M})$ for $1 \mathrm{hr}$ before imaging. Fluorescence intensity from the mushroom body lobes was monitored simultaneously at $520 \pm 15 \mathrm{~nm}$ for camgaroo emission and at $580 \pm 20$ and $660 \pm 20 \mathrm{~nm}$ for the ratiometric and dual-emitting fluor SNARF-1. At several times (arrowheads) while scanning, the SNARF-1loaded brains were treated with high-potassium solution ( $130 \mathrm{~mm}$ final) buffered to different $\mathrm{pH}$ values and containing the $\mathrm{H}^{+}$ionophore nigericin $(10 \mu \mathrm{g} / \mathrm{ml})$. The $\mathrm{pH}$ of the incubation medium was sampled at various times while scanning (arrows) to compare with the camgaroo and SNARF-1 fluorescence. $A$, Fluorescence of camgaroo-1 and the SNARF-1 660:580 ratio from two different brains with images collected once every $2.5 \mathrm{sec}$. At $\sim 6 \mathrm{~min}$ and every 8 min thereafter, the $\mathrm{pH}$ of the culture medium was adjusted. $B, A$ summary plot that illustrates the relationship between camgaroo-1 fluorescence, the SNARF-1 660:580 fluorescence ratio, and pH. Camgaroo-1 fluorescence increases with increasing intracellular pH. The SNARF-1 660:580 fluorescence ratio increases with increasing $\mathrm{pH}$. C, Fluorescence of camgaroo-2 and the SNARF-1 $660: 580$ ratio from two different brains with images collected once every $2.5 \mathrm{sec}$. At $\sim 6$ min and every 8 min thereafter, the $\mathrm{pH}$ of the culture medium was adjusted. D, A summary plot that illustrates the relationship between camgaroo-2 fluorescence, the SNARF-1 660:580 fluorescence ratio, and pH. Camgaroo-2 fluorescence also increases with increasing intracellular pH. As before, the SNARF-1 660:580 fluorescence ratio increases with increasing $\mathrm{pH}$. 
haps attributable to metabolic acidosis or the activation of $\mathrm{Ca}^{2+}-\mathrm{H}^{+}$exchangers (Zhan et al., 1998; Meyer et al., 2000; Xiong et al., 2000). Because the mechanism by which camgaroos sense $\mathrm{Ca}^{2+}$ is intrinsically sensitive to $\mathrm{pH}$ (Baird et al., 1999), we monitored the intracellular $\mathrm{pH}$ of mushroom body neurons during depolarization with the dual-emitting and ratiometric $\mathrm{pH}$ reporter SNARF-1 AM. The imaging was performed in the presence of the proton ionophore nigericin, so that adjustments made to the $\mathrm{pH}$ of the incubation medium in the initial experiments would also equilibrate the intracellular $\mathrm{pH}$.

Altering the intracellular $\mathrm{pH}$ of the mushroom body neurons in the isolated brains dramatically changes the fluorescence of camgaroo (Fig. 4). Figure 4, $A$ and $B$, illustrates an experiment with two different brains expressing camgaroo- 1 in which the intracellular $\mathrm{pH}$ was adjusted in steps from $\mathrm{pH} 7.06$, to 7.31, to 7.04 , to 7.89. Camgaroo-1 fluorescence increased with each step toward greater alkalinity and decreased with each step toward greater acidity. The $\mathrm{pH}$ alterations made to the incubation medium were reflected by the 660:580 emission ratio of SNARF-1, with the ratio increasing with increasing $\mathrm{pH}$. Parallel results were observed with camgaroo-2 in an experiment of similar design (Fig. 4C,D). Thus, both camgaroo- 1 and camgaroo- 2 exhibited the expected sensitivity to $\mathrm{pH}$. Furthermore, the fluorescence ratio of the intracellular indicator of $\mathrm{pH}$, SNARF-1, followed the changes in $\mathrm{pH}$ made to the incubation medium.

These results allowed us to follow the intracellular $\mathrm{pH}$ in mushroom body neurons along with camgaroo fluorescence while the mushroom body neurons were depolarized with high potassium. Brains expressing camgaroo in the mushroom bodies were loaded intracellularly with SNARF-1 and depolarized with potassium, and the fluorescence emissions were followed simultaneously to assay for the effect of depolarization on intracellular $\mathrm{pH}$ and camgaroo fluorescence. Camgaroo-1 fluorescence increased as expected with depolarization (Fig. 5A). However, the SNARF-1 ratio decreased slightly with depolarization in the representative brain trace shown in Figure 5A. This suggested that intracellular $\mathrm{pH}$ decreased slightly with depolarization. To determine whether this was generally the case, we followed the SNARF-1 ratio with depolarization in five separate brains (Fig. $5 B$ ). In all cases, the SNARF-1 emission ratio decreased slightly with depolarization. Parallel results were obtained with brains expressing camgaroo-2 (Fig. $5 C, D$ ). These results combined with those above indicate that depolarization produces an influx of extracellular calcium and a slight, but significant, increase in acidity. Most importantly, because camgaroo fluorescence decreases with increasing acidity (Fig. 4), these results indicate that the increased camgaroo fluorescence observed with depolarization cannot be attributable to changes in intracellular $\mathrm{pH}$.

\section{Neurotransmitter-induced increases in camgaroo fluorescence in the mushroom bodies}

We wondered whether the camgaroos could report on calcium influxes produced by a more natural stimulus (the presentation of neurotransmitter), in addition to the strong depolarization produced by elevating extracellular potassium. ACh is the putative excitatory neurotransmitter used by many of the neurons in the adult insect brain. The evidence for this is circumstantial; staining adult insect brains for the biosynthetic enzyme for $\mathrm{ACh}$, choline acetyltransferase, produces signal in much of the brain, including the antennal lobes and the antennocerebral tract (Kreissl and Bicker, 1989; Yasuyama and Salvaterra, 1999). The antennocerebral tract is the nerve that projects from the antennal lobe to the calyces of the mushroom body neurons (Roman and
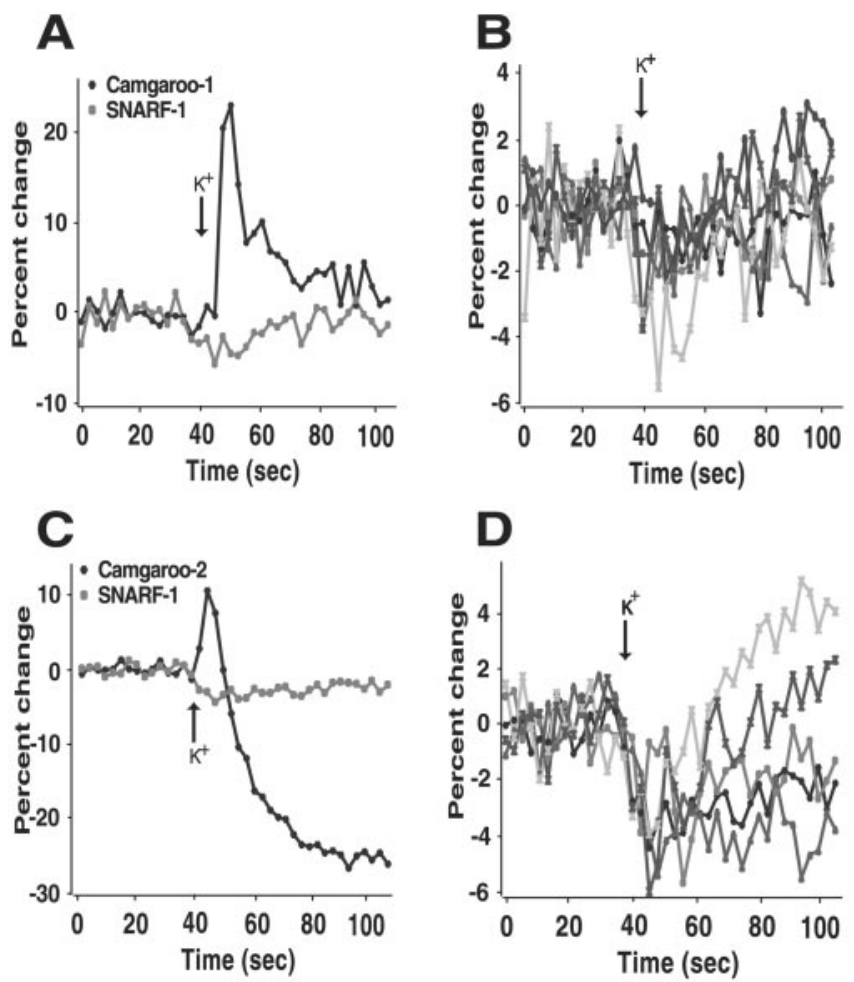

Figure 5. The $\mathrm{pH}$ changes induced by depolarization. Brains were incubated with the membrane-permeable $\mathrm{pH}$ indicator SNARF-1 $(50 \mu \mathrm{m})$ for $1 \mathrm{hr}$ before imaging. Fluorescence intensity from the mushroom body lobes was monitored simultaneously at $520 \pm 15 \mathrm{~nm}$ for camgaroo emission and at $580 \pm 20$ and $660 \pm 20 \mathrm{~nm}$ for the ratiometric and dual-emitting fluor SNARF-1. At $40 \mathrm{sec}$, brains were treated with high potassium (77 mm final), and the fluorescence change of camgaroo and SNARF- 1 was monitored. $A$, The traces from a representative brain expressing camgaroo- 1 in the mushroom bodies. There was a slight decrease in the 660:580 ratio of SNARF-1 fluorescence with depolarization but an increase in camgaroo- 1 fluorescence. $B$, A family of traces for five camgaroo-1-expressing brains loaded with SNARF-1. There was a slight decrease in the 660:580 fluorescence ratio for all brains with depolarization. C, The traces from a representative brain expressing camgaroo- 2 in the mushroom bodies. There was a slight decrease in the 660:580 ratio of SNARF-1 fluorescence with depolarization but an increase in camgaro0-2 fluorescence. D, A family of traces for five camgaroo-2-expressing brains loaded with SNARF-1. There was a slight decrease in the $660: 580$ fluorescence ratio with depolarization.

Davis, 2001). We therefore used pressure application of ACh through a puffer micropipette to stimulate the calyces of the mushroom bodies in brains expressing camgaroo (Fig. 6A). Application of ACh to only one area led to a response. This was an area limited to the region of cortex-neuropil that resides immediately anterior to the calyces in the brain. Pressure application of $\mathrm{ACh}$ to other areas of the adult brain in culture failed to produce any increase in fluorescence. For example, application to the cortical region just posterior to the mushroom bodies produced no elevation in signal.

Application of ACh to only one calyx produced a very rapid increase in fluorescence from the ipsilateral mushroom body lobes that peaked 1 or $2 \mathrm{sec}$ after neurotransmitter was applied. No response was detected in the cell body region from either hemisphere or in the lobe neuropil contralateral to the side of application (Fig. $6 \mathrm{~B}$ ). The lack of a camgaroo- 1 response in the cell bodies is interesting in light of their very robust response to potassium depolarization (Fig. $2 G$ ). These data together suggest that the cell bodies have voltage-dependent calcium channels that are required to activate camgaroo-1 (Figs. $2 G, 3 A, E$ ) with potassium depolarization, but neurotransmitter application fails to 
A
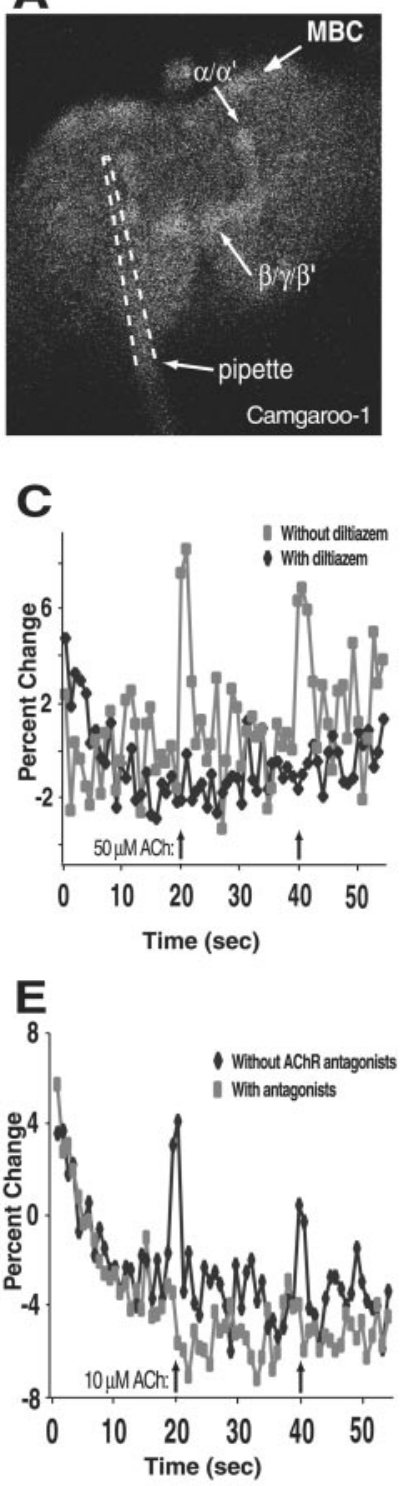
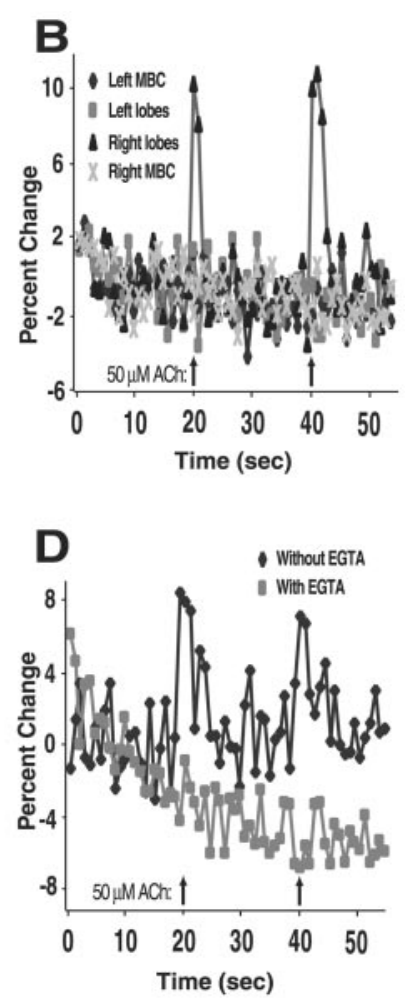

$\mathbf{F}$

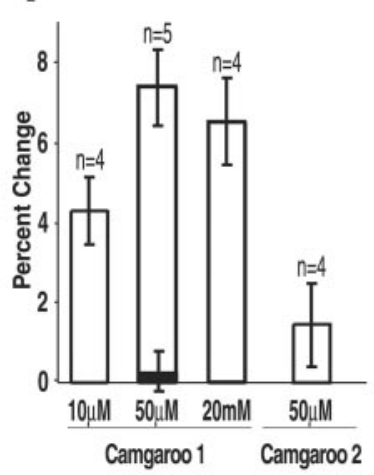

Figure 6. Localized application of ACh to the calyx of the mushroom body induces very rapid fluorescent responses of camgaroo-1. A illustrates a dissected brain expressing camgaroo-1 in the mushroom bodies before neurotransmitter application. The position of a micropipette used to deliver ACh locally to the calyx is shown in outline. MBC, Mushroom body neurons. B, Localized application of $50 \mu \mathrm{m}$ ACh to the right calyx induces calcium responses in the ipsilateral lobes but not in the contralateral lobes or cell bodies. Two $2 \mathrm{sec}$ pulses (arrows) of ACh were given, one at 20 sec into the scan and another at $40 \mathrm{sec}$. These traces are representative of five brains tested. C, Calcium responses induced by localized ACh application are blocked by inhibitors of voltage-gated calcium channels. Traces are representative of four brains that were tested. Each brain was firstestedfor AChresponsesipsilaterally without diltiazem at 20 and 40 secinto the scan. Diltiazem (100 $\mu$ m final) was added to the incubation medium, and each brain was retested $10-15$ min later. Two sequential scans of one brain are illustrated together on the same timeline. Diltiazem completely blocked the responses.D, Calciumresponses induced by localized AChapplication areblocked byextracellularEGTA. Traces are representative of four brains that were tested. Each brain was first tested for ACh responses ipsilaterally without EGTA at 20 and 40 sec into the scan. EGTA ( $6 \mathrm{~mm}$ final) was added to the incubation medium, and each brain was retested $10-15$ min later. Two sequential scans of one brain are illustrated together on the sametimeline. EGTA completely blocked the responses.E, Calcium responses induced by localized ACh application are blocked by antagonists of ACh receptors. Traces are representative of four brains that were tested. Each brain was first tested ipsilaterally for ACh responses without the antagonists at 20 and 40 sec into the scan. Antagonists ( $1 \mathrm{~mm}$ tubocurine and $40 \mu \mathrm{g} / \mathrm{ml}$ bungarotoxin) were added to the incubation medium, and the camgaroo- 1 responses wereretested $10-15$ min later. The two sequential scans of one brain are illustrated together on the same timeline. The antagonists completely blocked the responses. $F$, Summary of camgaroo-1 responses in the ipsilateral lobes elicited by different concentrations of $\mathrm{ACh}$. ACh at $50 \mu \mathrm{m}$ produced the maximal fluorescence increase of camgaroo-1. There was no response of the contralateral lobes with $50 \mu \mathrm{m}$ ACh shown as the black bar.

produce a detectable activation of these channels. This may be because the neurotransmitter-induced depolarization fails to enter the cell bodies on these unipolar neurons or perhaps because the actual increase in calcium concentration in the soma is less than in the neuropil attributable to differences in the surface-tovolume ratio. In addition, the ipsilateral and site specificity of application required to produce a response suggests that mushroom body neurons respond directly to ACh. To determine whether the neurotransmitter response requires the influx of calcium, we applied ACh to this region in the presence of diltiazem or EGTA (Fig. 6C,D). Both agents blocked the response to neurotransmitter, indicating that ACh produces an influx of extracellular calcium through voltage-dependent calcium channels. We also applied ACh in the presence of the nicotinic ACh receptor antagonists tubocurine and bungarotoxin. These antagonists fully blocked the ACh-induced response (Fig. $6 E$ ). Therefore, the application of neurotransmitter to the calyx of the mushroom bodies produces an influx of calcium as detected by camgaroo-1 through the stimulation of nicotinic acetylcholine receptors and voltage-dependent calcium channels. A concentration of $50 \mu \mathrm{M}$ ACh was saturating for this response (Fig. $6 F$ ).

\section{All types of mushroom body neurons respond to ACh}

The response of camgaroo to calcium in the mushroom bodies along with the high-resolution imaging of mushroom body neurons offered the opportunity to explore the calcium transients induced by ACh in the axons, dendrites, and cell bodies of different classes of mushroom body neurons. In other words, the imaging technology described above allowed us to determine for the first time whether all types of mushroom body neurons respond to ACh. Brains were mounted in culture standing upright but tilted slightly toward to posterior so that neurotransmitter could be applied to the anterior calyx (dendrites) and all lobes (axons) and cell bodies could be visualized (Fig. 7). At high magnification, such preparations (Fig. 7A) gave excellent resolution between the $\alpha$ and $\alpha^{\prime}$ lobes (Fig. 7C) and between the $\beta, \beta^{\prime}$, and $\gamma$ lobes (Fig. 7E).

The application of ACh to the calyx at two discrete times during imaging produced a significant fluorescence increase in the combined lobes (Fig. 7B). Quantitation of the image responses among the various lobes showed that the $\alpha$ and $\alpha^{\prime}$ lobes responded equally (Fig. $7 D$ ). Moreover, all of the horizontally oriented lobes, including the $\beta, \beta^{\prime}$, and $\gamma$ lobes, showed a rapid response that quickly returned to baseline (Fig. $7 F$ ). Therefore, these data, along with those in Figure 6, show that the axons of all mushroom body neurons, but not their cell bodies, respond to ACh stimulation. All classes of mushroom body neurons may therefore receive excitatory cholinergic input from the antennocerebral tract.

\section{Discussion}

Camgaroo transgenes for monitoring calcium levels

We demonstrated here that transgenes encoding the camgaroos can be used as effective sensors of intracellular calcium dynamics within the brain. Transgenic reporters have several advantages over the alternative of using calcium dyes. The reporters when expressed from specific promoters on transgenes provide for a limitless number of experimental animals with the identical pattern and a consistent level of reporter expression. In contrast, dyes need to be loaded into specific cells or the brain region of interest bathed with a cell-permeable dye. The latter method is disadvantageous because dyes have limited permeability and of- 

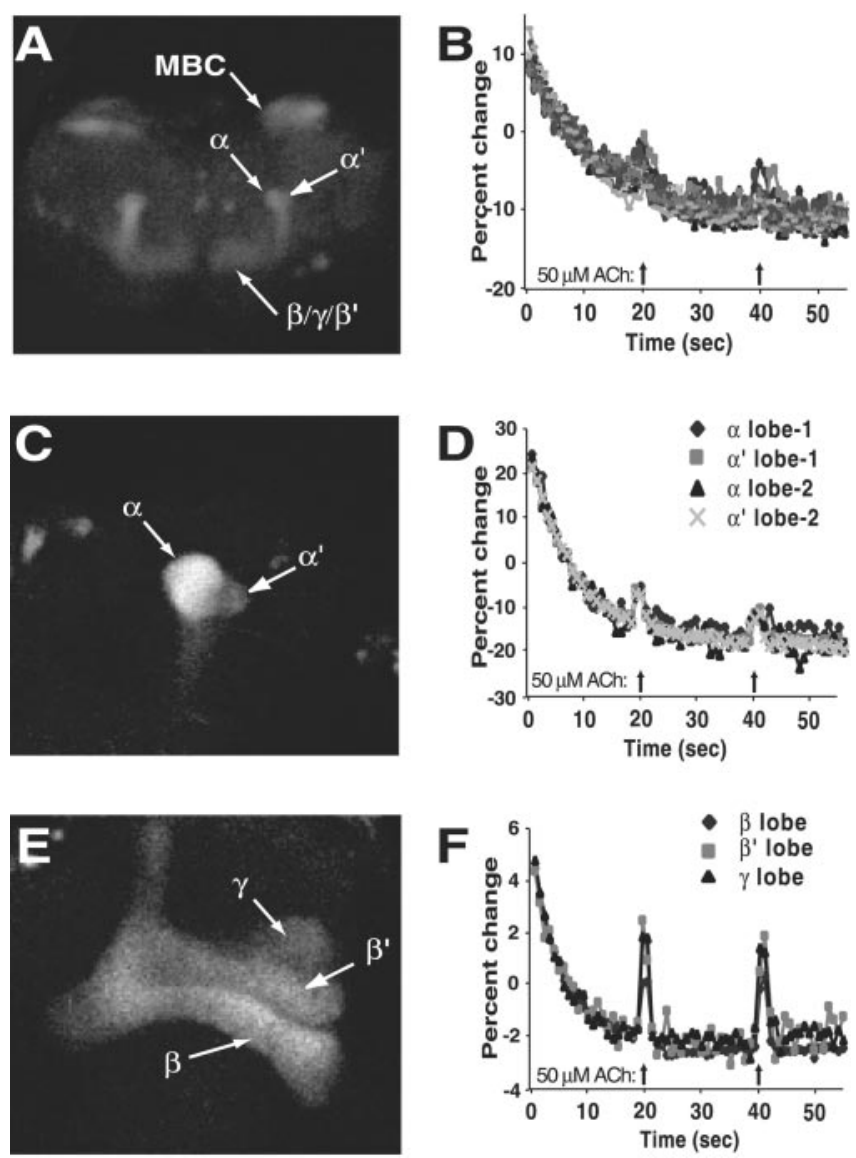

Figure 7. Local application of $\mathrm{ACh}$ to the anterior calyx produces calcium influx into all classes of mushroom body neurons as detected by camgaroo-2. A, A dorsofrontal view of a dissected brain expressing camgaroo- 2 in the mushroom bodies showing the cell bodies of mushroom body neurons $(M B C)$ and the $\alpha, \alpha^{\prime}, \beta, \beta^{\prime}$, and $\gamma$ lobes. The $\beta, \beta^{\prime}$, and $\gamma$ lobes are not distinguishable from each other at low magnification. $B$, The fluorescent responses in the combined lobes of five brains after two 2 sec pulses of $50 \mu \mathrm{m}$ ACh delivered to the calyx. Despite the rapid initial bleaching of camgaroo-2, the calcium responses in the lobes are evident. $C, A$ higher magnification of the $\alpha$ and $\alpha^{\prime}$ lobes of a brain before stimulation with ACh. In this view, the axon terminals of the $\alpha / \beta$ mushroom body neurons form a bright, spherical area of neuropil. The axon terminals of $\alpha^{\prime} / \beta^{\prime}$ neurons form the dimmer but spherical "bud" of neuropil adjacent to the $\alpha$ lobe. These areas were circumscribed and quantitated for response in $D$. D, The fluorescent responses in the $\alpha$ and $\alpha^{\prime}$ lobes of two brains pulsed with two 2 sec applications of $50 \mu \mathrm{m} A C h$ to the anterior calyx. The axons of neurons that populate both lobes respond. $E, A$ higher magnification of the $\beta, \beta^{\prime}$, and $\gamma$ lobes of a brain before being stimulated with $A C h$. The axons of $\alpha / \beta$ neurons form the lower "toe" of this image, those of $\alpha^{\prime} / \beta^{\prime}$ neurons form the middle "toe," and the $\gamma$ neurons form the dorsal, neuropil bud. These regions were quantitated for responses in $F$. F, The fluorescent responses in the $\beta, \beta^{\prime}$, and $\gamma$ lobes of a brain pulsed with two 2 sec applications of $50 \mu \mathrm{m} A C h$ to the calyx. The axons of neurons that populate all lobes show calcium influx with stimulation.

ten only penetrate into the superficial layer of cells. This prohibits imaging neurons that are in deep layers in the brain (Wang et al., 2001). To image calcium dynamics in axons and dendrites, sufficient dye needs to penetrate the neuron so that detectable quantities diffuse into the processes. The camgaroos used here diffuse freely into these processes in the Drosophila brain and allow for their visualization. A major advantage of the transgenic reporter approach using Drosophila is the availability of a near limitless collection of GAL4 drivers that offer the capability of expressing reporters in many different cell types (Brand and Perrimon, 1993; Yang et al., 1995). In addition, GAL4 drivers have been developed recently that offer control of both spatial and temporal patterns of reporter expression (Osterwalder et al., 2001; Roman et al., 2001).
Camgaroo-1 or camgaroo-2: which is best for you?

The camgaroo-2 reporter was selected as a brighter variant of camgaroo-1 (Griesbeck et al., 2001), and sequence analysis revealed a single change at residue 69 from glutamine to methionine. Although the two molecules are similar for their $K_{\mathrm{d}}$ values for calcium, dynamic range, and excitation and emission spectra (Baird et al., 1999; Griesbeck et al., 2001), they differ in several response properties as shown here. As expected, camgaroo-2 is the significantly brighter reporter with expression from the same driver (Fig. 1). This provides an advantage for some experiments in which it may be desirable to have basal expression to outline a region of interest. For example, camgaroo- 1 expression was too weak to adequately visualize and image the $\gamma, \beta$, and $\beta^{\prime}$ mushroom body lobes that are deep in the brain (Fig. 7; data not shown). However, camgaroo-2 has two unexpected disadvantages. The magnitude of the camgaroo- 2 response is less than camgaroo-1 (Figs. 2E-J, 6F), and the fluorescence of camgaroo-2 appears to quench or bleach more rapidly (Figs. $2 E-H, 7 B, D, F$ ). We assume that unknown and intrinsic biophysical differences in the two reporters attributable to the single amino acid change underlie these unexpected observations. Nevertheless, the differences between the reporters may provide greater flexibility for different experimental situations.

Calcium reporters similar to camgaroo have been constructed recently. One variant uses a circularly permuted enhanced GFP with an $\mathrm{M} 13$ peptide at the $\mathrm{N}$ terminus and calmodulin moiety at the $\mathrm{C}$ terminus (Nakai et al., 2001). This molecule has a $K_{\mathrm{d}}$ for calcium of $\sim 0.24 \mu \mathrm{M}$ and is therefore potentially more sensitive to small elevations in $\mathrm{Ca}^{2+}$ than either of the camgaroos. Nagai et al. (2001) constructed several analogous molecules known as pericams, which include versions that increase or decrease fluorescence or shift excitation wavelengths in response to $\mathrm{Ca}^{2+}$.

\section{GFPs and $\mathrm{pH}$}

A concern when using any GFP derivative as a biosensor is the $\mathrm{pH}$ sensitivity of these molecules. The camgaroos and other GFP derivatives are highly sensitive to $\mathrm{pH}$ (Griesbeck et al., 2001). To rule out the possibility that the change in fluorescence of the camgaroos was induced by $\mathrm{pH}$ changes, we monitored the $\mathrm{pH}$ intracellularly with SNARF-1 in the Drosophila mushroom body neurons during depolarization. The average change in fluorescence ratio of SNARF-1 across various $\mathrm{pH}$ values (Fig. 4) is $0.95 \%$ per $0.01 \mathrm{pH}$ units. The average drop in fluorescence ratio of $2-3 \%$ that occurs with potassium depolarization (Fig. $5 B, D$ ) suggests therefore that there is an acidification of mushroom body neuron cytoplasm of $\sim 0.02-0.03 \mathrm{pH}$ units with strong depolarization. The magnitude of this change is remarkably similar to that observed from spontaneous field bursts in the dentate gyrus (Xiong et al., 2000). These changes may be attributable to increased metabolic demands or an acidification that occurs from the increased activity of calcium-hydrogen transporters. Nevertheless, camgaroo fluorescence decreases with acidification, so the observed increase in camgaroo fluorescence with depolarization must be attributable primarily to a rise in $\mathrm{Ca}^{2+}$, slightly opposed by the small drop in $\mathrm{pH}$.

\section{All mushroom body neurons respond to acetylcholine}

One neurotransmitter delivered to mushroom body neurons from the antennocerebral tract is thought to be ACh. A primary reason for this hypothesis is that choline acetyltransferase, the synthetic enzyme for ACh, has been histochemically localized to this pathway (Yasuyama and Salvaterra, 1999). In addition, ACh evokes an increase in intracellular calcium in cultured mushroom 
body neurons from the adult cricket (Cayre et al., 1999) and the honeybee (Bicker, 1996). Moreover, electrical stimulation of the antennal lobe of the isolated honeybee brain evokes a postsynaptic response recorded from the mushroom bodies that is blocked by bath application of nicotinic ACh receptor antagonists (Oleskevich, 1999). These studies collectively support that idea that cholinergic pathways are important for the transmission of olfactory information from the antennal lobe to some mushroom body neurons, but they have not defined which types of mushroom body neurons are responsive to cholinergic transmission.

We used Drosophila brains expressing the camgaroos in the mushroom bodies to demonstrate that ACh application near the calyx evokes a rapid and ipsilateral-specific response in the mushroom body lobes. Moreover, the lobes representing all three classes of mushroom body neurons respond, consistent with the conclusion that all types of mushroom body neurons respond to cholinergic inputs. The definition of an excitatory neurotransmitter that can be used to stimulate mushroom body neurons will now permit studies on the effects of neuromodulators, such as dopamine or octopamine. Mushroom body neurons are known to express receptors for both types of neuromodulators (Han et al., 1996, 1998).

\section{References}

Augustine GJ (2001) How does calcium trigger neurotransmitter release? Curr Opin Neurobiol 11:320-326.

Baird GS, Zacharias DZ, Tsien RY (1999) Circular permutations and receptor insertion within green fluorescent proteins. Proc Natl Acad Sci USA 96:11241-11246.

Bicker G (1996) Transmitter-induced calcium signaling in cultured neurons of the insect brain. J Neurosci Methods 69:33-41.

Bootman MD, Collins TJ, Peppiatt CM, Prothero LS, MacKenzie L, DeSmet P, Travers M, Tovey SC, Seo JT, Berridge MJ, Ciccolini F, Lipp P (2001) Calcium signaling - an overview. Cell Dev Biol 12:3-10.

Boyarsky G, Ganz MB, Sterzel RB, Boron WF (1988) pH regulation in single glomerular mesangial cells. I. Acis extrusion in absence and presence of $\mathrm{HCO}^{3-}$. Am J Physiol 255:C844-C856.

Brand A, Perrimon N (1993) Targeted gene expression as a means of altering cell fates and generating dominant phenotypes. Development 118:401-415.

Cayre M, Buckingham SD, Yagodin S, Sattelle DB (1999) Cultured insect mushroom body neurons express functional receptors for acetylcholine, GABA, glutamate, octopamine, and dopamine. J Neurophysiol 81:1-14.

Chiesa A, Rapizzi E, Tosello V, Pinton P, DeVirgilio M, Fogarty KE, Rizzuto R (2001) Recombinant aequorin and green fluorescent protein as valuable tools in the study of cell signaling. Biochem J 355:1-12.

Crittenden J, Skoulakis EMC, Han K-A, Kalderon D, Davis RL (1998) Tripartite mushroom body architecture revealed by antigenic markers. Learn Mem 5:38-51.

Griesbeck O, Baird GS, Campbell RE, Zacharias DA, Tsien RY (2001) Reducing the environmental sensitivity of yellow fluorescent protein. J Biol Chem 276:29188-29194.

Han K-A, Millar NS, Groteweil MS, Davis RL (1996) DAMB, a novel dopamine receptor expressed specifically in Drosophila mushroom bodies. Neuron 16:1127-1135.

Han K-A, Millar NS, Davis RL (1998) A novel octopamine receptor with preferential expression in Drosophila mushroom bodies. J Neurosci $18: 3650-3658$.

Hockerman GH, Peterson BZ, Johnson BD, Catterall WA (1997) Molecular determinants of drug binding and action on L-type calcium channels. Annu Rev Pharmacol Toxicol 37:361-396.

Kerr R, Lev-Ram V, Baird G, Vincent P, Tsien RY, Schafer WR (2000) Optical imaging of calcium transients in neurons and pharyngeal muscle of C. elegans. Neuron 26:583-594.

Kreissl S, Bicker G (1989) Histochemistry of acetylcholinesterase and immunocytochemistry of an acetylcholine receptor-like antigen in the brain of the honeybee. J Comp Neurol 286:71-84.

Meyer TM, Munsch T, Pape HC (2000) Activity-related changes in intracellular $\mathrm{pH}$ in rat thalamic relay neurons. NeuroReport 11:33-37.

Miyawaki A, Llopis J, Heim R, McCaffery JM, Adams JA, Ikura M, Tsien RY (1997) Fluorescent indicators for $\mathrm{Ca}^{2+}$ based on green fluorescent proteins and calmodulin. Nature 388:882-887.

Morales M, Ferrus A, Mantinez-Padron M (1999) Presynaptic calciumchannel currents in normal and csp mutant Drosophila peptidergic terminals. Eur J Neurosci 11:1818-1826.

Nagai T, Sawano A, Park ES, Miyawaki A (2001) Circularly permuted green fluorescent proteins engineered to sense $\mathrm{Ca}^{2+}$. Proc Natl Acad Sci USA 98:3197-3202.

Nakai J, Ohkura M, Imoto K (2001) A high signal-to-noise $\mathrm{Ca}^{2+}$ probe composed of a single green fluorescent protein. Nat Biotech 19:137-141.

Oleskevich S (1999) Cholinergic synaptic transmission in insect mushroom bodies in vitro. J Neurophysiol 82:1091-1096.

Osterwalder T, Yoon KS, White BH, Keshishian H (2001) A conditional, tissue-specific expression system using inducible GAL4. Proc Natl Acad Sci USA 98:12596-12601.

Roman G, Davis RL (2001) Molecular biology and anatomy of Drosophila olfactory associative learning. BioEssays 23:571-581.

Roman G, He J, Davis RL (1999) New series of Drosophila expression vectors suitable for behavioral rescue. Biotechniques 27:54-56.

Roman G, Endo E, Zong L, Davis RL (2001) P\{Switch\}, a novel system for spatial and temporal control of gene expression in Drosophila melanogaster. Proc Natl Acad Sci USA 98:12602-12607.

Rose CR, Konnerth A (2001) Stores not just for storage: intracellular calcium release and synaptic plasticity. Neuron 31:519-522.

Stewart BA, Atwood HL, Renger JJ, Wang J, Wu CF (1994) Improved stability of Drosophila larval neuromuscular preparations in the haemolymph-like physiological solutions. J Comp Physiol 175:179-191.

Wang Y, Wright NJD, Guo H-F, Xie Z, Svoboda K, Malinow R, Smith DP, Zhong Y (2001) Genetic manipulation of the odor-evoked distributed neural activity in the Drosophila mushroom body. Neuron 29:267-276.

West AE, Chen WG, Dalva MB, Dolmetsch RE, Kornhauser JM, Shaywitz AJ, Takasu MA, Tao X, Greenberg ME (2001) Calcium regulation of neuronal gene expression. Proc Natl Acad Sci USA 98:11024-11031.

Wicher D, Walther C, Wicher C (2001) Non-synaptic ion channels in insects-basic properties of currents and their modulation in neurons and skeletal muscles. Prog Neurobiol 64:431-525.

Xiong Z-Q, Saggau P, Stringer JL (2000) Activity-dependent intracellular acidification correlates with the duration of seizure activity. J Neurosci 20:1290-1296.

Yang M-Y, Armstrong JD, Vilinsky I, Strausfeld NJ, Kaiser K (1995) Subdivision of the Drosophila mushroom bodies by enhancer-trap expression patterns. Neuron 15:45-54.

Yasuyama K, Salvaterra PM (1999) Localization of the choline acetyltransferase-expressing neurons in Drosophila nervous system. Microsc Res Tech 45:65-79.

Yuste R, Denk W (1995) Dendritic spines as basic functional units of neuronal integration. Nature 375:682-684.

Zhan RZ, Fujiwara N, Tanaka E, Shimoi K (1998) Intracellular acidification induced by membrane depolarization in rat hippocampal slices: roles of intracellular $\mathrm{Ca}^{2+}$ and glycolysis. Brain Res 780:86-94.

Zucker RS (1999) Calcium-and activity-dependent synaptic plasticity. Curr Opin Neurobiol 9:305-313. 